\title{
COVID-19 associated collapsing glomerulopathy presenting as acute kidney injury on chronic kidney disease: a case report and review of the literature
}

\author{
Onoriode Kesiena ${ }^{1}$ (D . Pia Papadopoulos ${ }^{1} \cdot$ Dominic Amakye ${ }^{1} \cdot$ Eunice Hama $^{1} \cdot$ Rene Mackay $^{2}$
}

Received: 26 August 2021 / Accepted: 17 November 2021 / Published online: 26 November 2021

(C) Japanese Society of Nephrology 2021

\begin{abstract}
Traditionally collapsing glomerulopathy (CG) is associated with medications, autoimmune disease, viral infection and the APOL1 gene variant seen in blacks/African Americans. Most reported cases of acute kidney injury (AKI) in COVID-19 infected individuals have been in individuals without prior CKD. In this report, we present a 49-year-old African American female with a past medical history of chronic kidney disease (CKD) stage 4, hypertension, type 2 diabetes mellitus, recent COVID-19 infection, and a repeat positive blood test for COVID-19 more than 21 days after the initial result, who presented with an AKI on CKD. Renal biopsy revealed a collapsing glomerulopathy. She was started on hemodialysis and did not receive immunosuppressive therapy due to the advanced scaring seen on the renal biopsy. Concerning the pathophysiology of COVID-19-associated CG, researchers have postulated different mechanisms such as a direct cytopathic effect of the virus on podocytes, immune dysregulation, and fluid imbalance. This is one of a few cases of AKI on CKD due to CG related to COVID-19. The mechanism of CG was, however, unclear. Currently, there is no specific interventions to prevent the development of CG in patients with COVID-19 infection. Further studies should investigate measures to prevent the development of CG.
\end{abstract}

Keywords COVID-19 $\cdot$ Collapsing $\cdot$ Glomerulopathy $\cdot$ Acute $\cdot$ Kidney $\cdot$ Injury

\section{Introduction}

COVID-19 related acute kidney injury (AKI) occurs due to an injury to renal tubular and or renal glomerulus [1-3]. This is supported by reported histopathological findings from living and postmortem kidneys that have shown features of tubular and or glomerular disease such as collapsing glomerulopathy (CG) [4]. Patients who experience such renal injuries may present with hematuria, proteinuria, oliguria, or elevated blood pressures [5]. Cases of COVID-19 infectionrelated CG in individuals who had traditional risk factors without any prior kidney disease have been reported. This

Onoriode Kesiena

onoriode.kesiena@piedmont.org

1 Department of Internal Medicine, Piedmont Athens Regional Medical Center, 1199 Prince Avenue, Athens, GA 30606, USA

2 Department of Nephrology, Piedmont Athens Regional Medical Center, Athens, GA, USA report serves to increase the awareness 'of COVID-19 associated CG in patients with prior kidney disease who present with an AKI on chronic kidney disease (CKD).

\section{Case report}

A 49-year-old African American female with a past medical history of preserved ejection heart failure, chronic kidney disease (CKD) stage 4, hypertension, type 2 diabetes mellitus, morbid obesity, and recent COVID-19 infection ( 2 weeks prior), was admitted with worsening bipedal swelling and reduced urine production. Despite adherence to medications, she admitted to elevated home blood pressure readings, weight gain, and exertional dyspnea. Home medications included Aspirin, Atorvastatin, Imdur, Metoprolol, Furosemide, Nifedipine, Potassium chloride, and Magnesium citrate.

On physical examination, she looked ill, blood pressure 151/106 mmHg, pulse 91 regular rate and rhythm, respiratory rate 18 , axillary temperature $97.7^{\circ} \mathrm{F}$, and 
Table 1 Basic metabolic profile during admission

\begin{tabular}{lllll}
\hline & Day 0 & Day 2 & Day 5 & Day 7 \\
\hline Sodium (mmol/L) & 141 & 142 & 141 & 133 \\
Potassium (mmol/L) & 4.1 & 3.9 & 3.9 & 3.6 \\
Chloride (mmol/L) & 108 & 108 & 102 & 96 \\
Bicarbonate (mmol/L) & 22 & 22 & 25 & 23 \\
BUN (mg/dL) & 49 & 49 & 66 & 40 \\
Creatinine (mg/dL) & 6.07 & 5.94 & 7.17 & 6.20 \\
BUN/Creatinine Ratio & 8 & 8 & 9 & 6 \\
Glucose (mg/dL) & 102 & 93 & 100 & 101 \\
Calcium (mg/dL) & 8.9 & 8.3 & 8.8 & 8.0 \\
\hline
\end{tabular}

Table 2 Urinalysis on admission, day 0

\begin{tabular}{ll}
\hline Color & Yellow \\
\hline Clarity & Hazy \\
Specific gravity & 1.015 \\
$\mathrm{pH}$ & 6.0 \\
Glucose & $1+$ \\
Ketones & $1+$ \\
Blood & Negative \\
Nitrite & Negative \\
Protein & $3+$ \\
Bilirubin & Negative \\
Urobilinogen & $<2.0$ \\
Leukocytes & Negative \\
RBC & $5 /$ high power field \\
WBC & $6 /$ high power field \\
Bacteria & None \\
Mucus & Few \\
Epithelial cells & $4 /$ high power field \\
\hline
\end{tabular}

oxygen saturation of $94 \%$ on room air. BMI $67.47 \mathrm{~kg} / \mathrm{m}^{2}$ (weight $-26.7 \mathrm{~kg}$, height $-1.75 \mathrm{~m}$ ) on admission and BMI $61.4 \mathrm{~kg} / \mathrm{m}^{2}$ (weight-188, height-1.75 m) before admission. The rest of the physical examination was unremarkable aside from bilateral pitting lower extremity edema and mild bibasal crackles. Laboratory investigations showed a white blood count of 8000 cells $/ \mu 1$, hemoglobin of $11.8 \mathrm{~g} / \mathrm{dl}$, and a basic metabolic profile as shown on day 0 in Table 1 . The patient's baseline Creatinine was between $2.2 \mathrm{mg} / \mathrm{dL}$ and $2.4 \mathrm{mg} / \mathrm{dL}$. Serum total protein $6.8 \mathrm{~g} / \mathrm{dL}$, Albumin $2.9 \mathrm{~g} / \mathrm{dL}$ and normal AST \& ALT. BNP was normal and HbA1c was $6.6 \%$. Urinalysis is as shown in Table 2. Chest $\mathrm{x}$-ray revealed mild interstitial edema, stable cardiomegaly with central vascular congestion. EKG showed left ventricular hypertrophy. Renal ultrasound showed no hydronephrosis, both kidneys measured $10 \mathrm{~cm}$ in length each and appeared echogenic.

The patient's care was multi-disciplinary and involved the hospitalist service, cardiology, and nephrology teams. On day 1 , her urine output was $800 \mathrm{ml} / 24-\mathrm{h}$ and are diuretic was held. She received a 1 liter slow normal saline fluid challenge with no improvement in her renal function as shown on day 2 in Table 1 . The fluid challenge was discontinued as the creatinine level did not improve. Further workup for AKI showed a fractional excretion of Urea $58.4 \%$, consistent with intrinsic kidney disease. Urine studies showed nephrotic range proteinuria, $19 \mathrm{~g} / \mathrm{g}$ (the previous study 2 years ago was $2.9 \mathrm{~g} / \mathrm{g}$ ). Serology workup showed a negative HIV test, ANA, anti-GBM, anti-DS DNA, ANCA, and cryoglobulins. $\mathrm{C} 3$ \& C4 were elevated at $270 \mathrm{mg} / \mathrm{dL}$ and $83 \mathrm{mg} / \mathrm{dL}$, respectively. A repeat COVID test was positive ( $>21$ days after the first positive test) with an elevated D-dimer of $>7650 \mathrm{ng}$ / $\mathrm{mL}$. A kidney biopsy was also performed, with the report presented below as shown in Fig. 1a-d (a-Biopsy of the kidney showing a collapsing lesion with microcystic tubules; b-Biopsy of the kidney with a silver stain showing a collapsing lesion; c-Biopsy of the kidney showing interstitial fibrosis under low power; $\mathrm{d}$-Biopsy of the kidney showing tubular injury with a collapsing lesion). Despite the removal of all nephrotoxic medications, the patient's kidney function continued to decline as shown on day 5. On account of this continued decline and the refractory fluid overload, with the associated uremic symptoms, she was started on renal replacement therapy through a right internal jugular permcath. Later on, she was discharged to continue hemodialysis as an outpatient since her renal function failed to recover. She did not receive any immunosuppressive therapy due to the extensive scaring renal scarring seen on biopsy.

\section{Kidney biopsy report}

The kidney biopsy images are as shown in Fig. 1a-d. On light microscopy, sections revealed cores of renal tissue, approximately $80 \%$ of which consist of the renal cortex. Thirty-three glomeruli were present, 19 of which were globally sclerotic. Five glomeruli showed segmental areas of capillary loop collapse and sclerosis with overlying podocyte hypertrophy and hyperplasia. Acute tubular injury was present. Moderate numbers of tubules show microcystic dilatation. A background of moderate interstitial fibrosis and tubular atrophy involved an estimated $40 \%$ of the submitted parenchyma. Prominent interstitial inflammation is present. There was severe fibrous intimal thickening of small arteries and severe hyalinosis of arterioles. A congo red stain for amyloid was negative. Examination of two Toluidine blue standard sections prepared for electron microscopy revealed areas of renal cortex containing one globally sclerotic glomerulus. On immunofluorescence, the sections were stained for IgG, IgM, IgA, C3, C1q, albumin, fibrinogen, and kappa and lambda light chains. The renal parenchyma submitted consisted of $30 \%$ cortex. Eight glomeruli were presented for evaluation with four globally sclerotic. There was no 


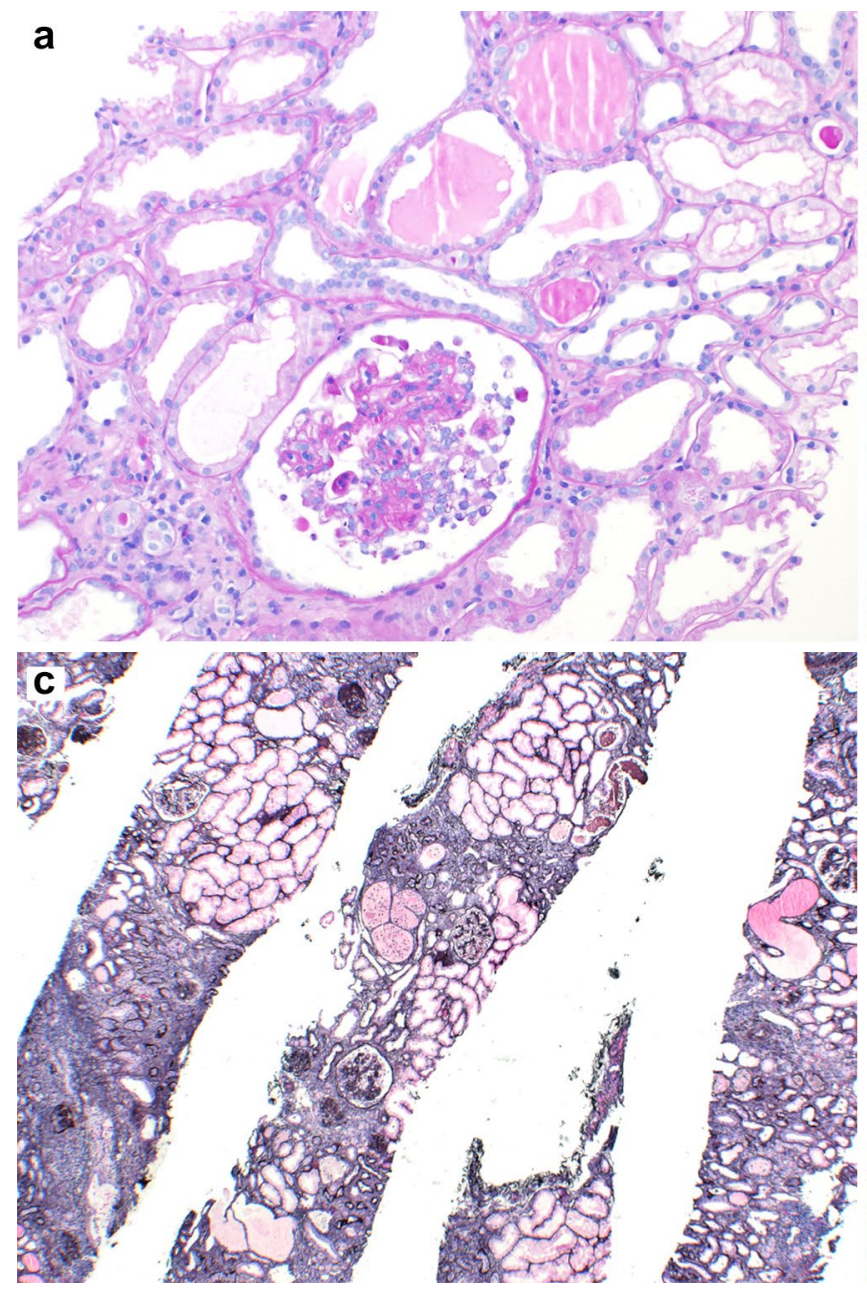

Fig. 1 a Biopsy of the kidney showing a collapsing lesion with microcystic tubules. b Biopsy of the kidney with a silver stain showing a collapsing lesion. c Biopsy of the kidney showing interstitial

significant extraglomerular staining. Kappa and lambda stained equally throughout the tubulointerstitium. Ultrastructural examination by electron microscopy was not done as the two prepared blocks showed no intact glomeruli.

\section{Discussion}

In this report, we described a case of collapsing glomerulopathy associated with SARS-CoV-2 infection, which presented as an AKI on CKD. This was an African American patient with morbid obesity, heart failure with preserved ejection fraction, and CKD. The CKD had been attributed to diabetes mellitus and or hypertension.

Research has shown that AKI with electrolyte abnormalities is a complication of SARS-CoV-2 infection [1, 6 , 7]. The pathophysiology of AKI associated with COVID19 infection includes fluid imbalance leading to a prerenal

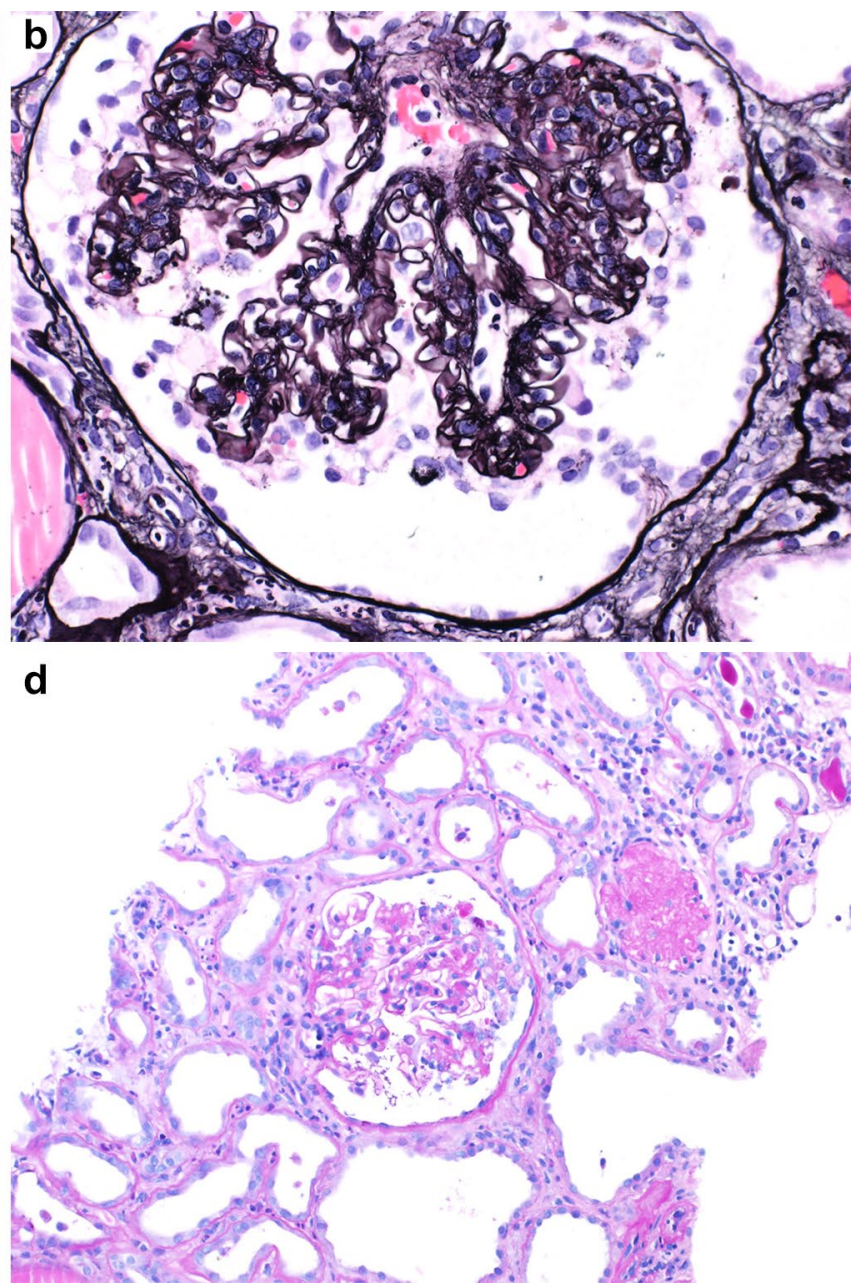

fibrosis under low power. d Biopsy of the kidney showing tubular injury with a collapsing lesion

AKI, toxic tubular damage following cytokine release, and a direct cytopathic effect of the virus $[8,9] 10$. One of the common histopathological findings in these acute kidney injuries is collapsing glomerulopathy. A glomerulopathy that has been associated with viral infections, autoimmune conditions, and medications [11, 12]. It has also been associated with a variant of the apolipoprotein L1 (APOL1) gene seen in blacks [13].

Post-mortem kidney biopsies via immunohistochemistry have identified viral proteins from SARS-CoV-2 RNA infection [14]. This has been equated as evidence of a cytopathic effect of the SARS-CoV-2 RNA virus on podocytes. It is postulated that the virus gains entry into the cells using the angiotensin-converting enzyme II (ACEII) receptor [15] 16. A receptor that is found in the capillary network of alveoli and glomeruli epithelial cells $[17,18]$. Unfortunately, the extensive glomeruli scarring in this patient's biopsy meant immunohistochemistry for evidence of SARS-CoV-2 
proteins or RNA could not be done, which makes it difficult to attribute the mechanism of injury in this case to a direct cytopathic effect. Kidney biopsies have also shown a microcystic tubular dilatation which was seen in this patient's biopsy, similar to lesions associated with variants of APOL1 that increases the risk of CG $[19,20]$.

A dysregulated immune response occurs with SARSCoV-2 infection similar to that seen in hemophagocytic syndrome [21]. With SARS-CoV-2 infection, an elevated level of several cytokines has been seen in both asymptomatic and symptomatic individuals [22,23]. This patient came in asymptomatic but later developed a fever, tachycardia with an elevated D-dimer in the second week of admission, with a positive COVID-19 test more than 21 days after her initial result. Thus, elevated levels of cytokines may have contributed to the CG.

With these mechanisms of injury in mind, one other factor that may have contributed to the rapid decline in her renal function is obesity. Obesity has been reported as one of the demographic factors associated with an AKI in patients who are infected with SARS-CoV-2. In a retrospective study of 773 patients with COVID-19 infection, morbid obesity was significantly associated with an increased risk of AKI [24]. Interesting our patient also was morbidly obese and dysregulation of fatty acid and carbohydrate metabolism, oxidative stress and inflammation are pathogenic factors that may have contributed to the AKI on CKD [25].

In conclusion, this case report highlights an acute on chronic kidney disease due to SARS-CoV-2 related glomerulopathy. Although the mechanism of injury remains uncertain, it should be suspected in asymptomatic COVID19 infected patients who present with an AKI on CKD or a rapidly worsening renal function especially if they are morbidly obese. To date, there is no specific intervention to prevent the development of CG in patients with COVID-19 infection however for the prevention of AKI, the consensus report of the 25th acute disease quality initiative (ADQI) workgroup recommend adequate fluid and hemodynamic management, and avoidance of nephrotoxic drug exposure where possible.

Author contributions OK MD, MPH: Introduction, discussion, case report. PP MD: Introduction and abstract. NK MD: Case report and abstract. DA MD: Discussion and case report. EH MD: Introduction, case report and discussion.

Funding We have no funding to declare.

Data availability Not applicable.

\section{Declarations}

Conflict of interest The authors have no conflicts of interest.
Ethical approval Not applicable.

Informed consent Informed consent was obtained from the patient in this report.

Consent to participate and publication Consent was obtained from the patient.

\section{References}

1. Incidence of acute kidney injury in COVID-19 infection: a systematic review and meta-analysis I SpringerLink [Internet]. Published: 16 June 2020 Available from: https://doi.org/10.1186/s13054-02003009-y. Accepted 4 Mar 2021

2. Ahmed AR, Ebad CA, Stoneman S, Satti MM, Conlon PJ. Kidney injury in COVID-19. WJN. 2020;9:18-32.

3. Batlle D, Soler MJ, Sparks MA, Hiremath S, South AM, Welling PA, et al. Acute kidney injury in COVID-19: emerging evidence of a distinct pathophysiology. JASN. 2020;31:1380-3.

4. Kudose S, Batal I, Santoriello D, Xu K, Barasch J, Peleg Y, et al. Kidney biopsy findings in patients with COVID-19. JASN. 2020;31:1959-68.

5. Larsen CP, Bourne TD, Wilson JD, Saqqa O, Sharshir MA. Collapsing glomerulopathy in a patient with COVID-19. Kidney Int Rep. 2020;5:935-9.

6. Yang X, Tian S, Guo H. Acute kidney injury and renal replacement therapy in COVID-19 patients: a systematic review and meta-analysis. Int Immunopharmacol. 2021;90:1059.

7. Zhou S, Xu J, Xue C, Yang B, Mao Z, Ong ACM. Coronavirusassociated kidney outcomes in COVID-19, SARS, and MERS: a meta-analysis and systematic review. Ren Fail. 2021;43:1-15.

8. Ronco C, Reis T. Kidney involvement in COVID-19 and rationale for extracorporeal therapies. Nat Rev Nephrol. 2020;16:308-10.

9. Hirsch JS, Ng JH, Ross DW, Sharma P, Shah HH, Barnett RL, et al. Acute kidney injury in patients hospitalized with COVID-19. Kidney Int. 2020;98:209-18.

10. Puelles VG, Lütgehetmann M, Lindenmeyer MT, Sperhake JP, Wong MN, Allweiss L, et al. Multiorgan and renal tropism of SARS-CoV-2. N Engl J Med. 2020;383:590-2.

11. Albaqumi M, Barisoni L. Current views on collapsing glomerulopathy. JASN. 2008;19:1276-81.

12. Larsen CP, Beggs ML, Saeed M, Walker PD. Apolipoprotein L1 risk variants associate with systemic lupus erythematosus-associated collapsing glomerulopathy. JASN. 2013;24:722-5.

13. Morales E, Alonso M, Gutiérrez E. Collapsing glomerulopathy: update. Medicina Clínica (Engl Ed). 2019;152:361-7.

14. Su H, Yang M, Wan C, Yi L-X, Tang F, Zhu H-Y, et al. Renal histopathological analysis of 26 postmortem findings of patients with COVID-19 in China. Kidney Int. 2020;98:219-27.

15. Multiorgan and renal tropism of SARS-CoV-2 I NEJM [Internet]. August 6, 2020 Available from: https://doi.org/10.1056/NEJMc 2011400. Accepted 8 Mar 2021

16. Li W, Moore MJ, Vasilieva N, Sui J, Wong SK, Berne MA, et al. Angiotensin-converting enzyme 2 is a functional receptor for the SARS coronavirus. Nature. 2003;426:450-4.

17. Bombardini T, Picano E. Angiotensin-converting enzyme 2 as the molecular bridge between epidemiologic and clinical features of COVID-19. Can J Cardiol. 2020;36:784.e1-784.e2.

18. Ye M, Wysocki J, William J, Soler MJ, Cokic I, Batlle D. Glomerular localization and expression of angiotensin-converting enzyme 2 and angiotensin-converting enzyme: implications for albuminuria in diabetes. JASN. 2006;17:3067-75. 
19. Collapsing glomerulopathy: a 30-year perspective and single, large center experience I Clinical Kidney Journal I Oxford Academic [Internet]. Available from: https://academic.oup.com/ckj/ article/10/4/443/3806647?login=true. Cited 9 Mar 2021

20. Larsen CP, Beggs ML, Saeed M, Ambruzs JM, Cossey LN, Messias NC, et al. Histopathologic findings associated with APOL1 risk variants in chronic kidney disease. Mod Pathol. 2015;28:95-102.

21. Kissling S, Rotman S, Gerber C, Halfon M, Lamoth F, Comte D, et al. Collapsing glomerulopathy in a COVID-19 patient. Kidney Int. 2020;98:228-31.

22. Han H, Ma Q, Li C, Liu R, Zhao L, Wang W, et al. Profiling serum cytokines in COVID-19 patients reveals IL-6 and IL-10 are disease severity predictors. Emerg Microbes Infect. 2020;9:1123-30.

23. Tjan LH, Furukawa K, Nagano T, Kiriu T, Nishimura M, Arii $\mathrm{J}$, et al. Early differences in cytokine production by severity of
Coronavirus Disease 2019. J Infect Dis. 2021. https://doi.org/10. 1093/infdis/jiab005.

24. Martín-del-Campo F, Ruvalcaba-Contreras N, Velázquez-Vidaurri AL, Cueto-Manzano AM, Rojas-Campos E, Cortés-Sanabria L, et al. Morbid obesity is associated with mortality and acute kidney injury in hospitalized patients with COVID-19. Clin Nutr ESPEN. 2021;45:200-5.

25. Schiffl H, Lang SM. Obesity, acute kidney injury and outcome of critical illness. Int Urol Nephrol. 2017;49:461-6.

Publisher's Note Springer Nature remains neutral with regard to jurisdictional claims in published maps and institutional affiliations. 